\title{
An Experimental Report on Correlated-Photon Ellipsometry
}

\author{
Kimani C. Toussaint, Jr. ${ }^{a}$, Giovanni Di Giuseppe ${ }^{a}$ \\ Alexander V. Sergienko ${ }^{a, b}$, Bahaa E. A. Saleh ${ }^{a}$, and Malvin C. Teich ${ }^{a, b}$ \\ ${ }^{a}$ Quantum Imaging Laboratory, Department of Electrical \& Computer Engineering \\ Boston University, Boston, MA, USA 02215-2421 \\ ${ }^{b}$ Department of Physics, Boston University, Boston, MA, USA 02215-2421
}

\begin{abstract}
Traditional ellipsometric measurements are limited in their accuracy because of the use of an external reference sample for calibration, and because of the noise inherent in the source at low light levels. We demonstrate that these limitations can be circumvented by using a non-classical source of light, namely, twin photons generated by the process of spontaneous parametric downconversion, in conjunction with a novel polarization interferometer and coincidence-counting detection scheme. The twin-photon nature of the source is a unique feature of our scheme. We are guaranteed, on the detection of a photon in one of the arms of the setup, that its twin will be in the other. We present experimental results showing how the technique operates.
\end{abstract}

Keywords: Ellipsometry, quantum optics, nonlinear optics

\section{INTRODUCTION}

The approach of using light to measure the properties of physical systems is the time-honored technique of optical metrology. Light emitted from a source is directed to the system whereupon it is reflected, transmitted, or scattered before reaching a detector. As long as the properties of the light are well characterized at the input and output of the system, one can infer information about the system. Furthermore, one can utilize the polarization degree of freedom of the light to obtain the optical constants of the system, a metrological approach known as ellipsometry. ${ }^{1-6}$ However, for reflection ellipsometry, for example, any time variation of the intensity of the light at the input of the system reduces the accuracy of the measurements made using this approach. Conventional (classical) ellipsometric procedures employ either interferometry using a beam splitter, or nulling, in order to mitigate this problem. In the case of the latter method, a standard reference sample is always used for the purposes of calibration, and hence this becomes the limit on the accuracy of the measurements. In the case of the former method, the problems stemming from the quantum noise of the light source, as well as the additional noise imparted by the beam splitter, become pronounced at low light levels. ${ }^{7}$ The accuracy of the measurements made in this scheme are limited by these noise fluctuations. These problems in accuracy, encountered by both the interferometric and nulling techniques of ellipsometry, are obviated in quantum ellipsometry. ${ }^{8,9}$ In short, quantum ellipsometry allows for accurate ellipsometric measurements to be obtained at low light levels without referencing to a standard sample, in spite of a time-varying source intensity.

We have previously shown elsewhere how quantum ellipsometry offers the possibility of achieving accurate ellipsometric measurements by utilizing a particular non-classical source of light, namely, twin photons generated by the process of spontaneous parametric downconversion (SPDC). Such a source has been used in many applications such as quantum teleportation, ${ }^{10}$ quantum cryptography, ${ }^{11}$ and the emerging field of quantum metrology. ${ }^{12,13}$ In this paper, we will first identify the desired attributes of an accurate ellipsometric measurement. Next, we will show how classical ellipsometry falls short of meeting these attributes. The special cases of null and interferometric ellipsometry will be discussed. Then, we will demonstrate how quantum ellipsometry, using correlated-photon pairs (also referred to as correlated-photon ellipsometry) meets these desired attributes. Finally, we will present results from an experiment on a silicon ( $\mathrm{Si}$ ) sample using correlated-photon ellipsometry.

Further author information: (Send correspondence to A.V.S.)

A.V.S.: E-mail: alexserg@bu.edu, Telephone: 1617353 6564, http://www.bu.edu/qil

Address: Boston University, Electrical \& Computer Engineering Department, 8 Saint Mary's Street, Boston, MA, USA $02215-2421$ 


\section{DESIRED ATTRIBUTES OF AN ACCURATE ELLIPSOMETRIC MEASUREMENT}

In this section we present four desired attributes of an accurate ellipsometric measurement that we have identified in a reflective configuration. The first, and by far the most important, is that no external reference sample be used for calibration. The accuracy of the measurements made on a sample after calibration by an external reference is limited to how well the reference has been characterized. This becomes especially problematic if the quality of the reference degrades with time. In addition, the best reference samples are only certified at specific angles of incidence and at a specific wavelength. ${ }^{3}$ The second attribute is that only $\mathrm{SU}(2)$ polarization elements and polarizers be used in the apparatus. Such SU(2) elements consist of a polarization rotator sandwiched between two wave retarders, and can perform any general unitary operation. The $2 \times 2$ Jones matrix representation for such an operation is given by

$$
\mathbf{T}_{S U(2)}=\left(\begin{array}{cc}
\cos \theta e^{i(\phi+\alpha)} & -\sin \theta e^{i(\phi-\alpha)} \\
\sin \theta e^{-i(\phi-\alpha)} & \cos \theta e^{-i(\phi+\alpha)}
\end{array}\right)
$$

where $\theta$ represents the angle of the axis of a polarization rotator with respect to the horizontal direction, while $\phi$ and $\alpha$ represent the retardation imparted by wave retarders. We do not include the general class of $\mathrm{SU}(2)$ elements because it would include beam splitters which are sources of noise at low light levels. The use of any other element, such as mirrors, would prevent one from separating the parameters of the system under test from the properties of these elements. The third attribute of an accurate ellipsometric measurement is for all measurements to be basis independent, e.g., that they not depend on the absolute azimuthal position of the polarizers. The fourth and final desired attribute calls for complete independence of source and detector characterization. This is important for any metrology setting that relies on measuring optical intensity.

\section{A GENERAL DESCRIPTION OF CLASSICAL ELLIPSOMETRY}

Consider a source that emits light of a single frequency but with an intensity, $I_{\text {in }}(t)$, that varies randomly in time. This light passes through a linear polarizer, whose transmission axis is fixed along the horizontal direction, before traversing a general unitary element which behaves as described in the previous section. The light then reflects from a sample, goes through a second general unitary element, and finally a polarization analyzer, oriented parallel to the first polarizer, before reaching an optical detector with quantum efficiency $\eta$. The Jones matrix for a linear polarizer whose transmission axis is fixed along the horizontal direction is given by

$$
\mathbf{T}_{p}=\left(\begin{array}{ll}
1 & 0 \\
0 & 0
\end{array}\right)
$$

while the action of the sample can be described as follows:

$$
\mathbf{T}_{s}=\left(\begin{array}{ll}
\tilde{r}_{11} & \tilde{r}_{12} \\
\tilde{r}_{21} & \tilde{r}_{22}
\end{array}\right)
$$

where $\tilde{r}_{m n}(m, n=1,2)$ is the complex reflection coefficient of the sample. The indices refer to the polarization modes of the beam impinging on, and reflecting from, the sample. For example, $\tilde{r}_{12}$ is the complex reflection coefficient corresponding to a beam polarized along mode 1 that is reflected polarized along mode 2 . For most ellipsometry applications, it is assumed the sample has known eigenpolarizations such that $\tilde{r}_{12}=\tilde{r}_{21}=0$.

This system is shown in Fig. 1 . The Jones vector at the detector, $\hat{\mathbf{J}}_{o}$, and hence the intensity, can be determined by treating this system as a multiplication of Jones matrices. If the state of the light after the first polarizer is given by the Jones vector $\hat{\mathbf{J}}_{i}$, then $\hat{\mathbf{J}}_{o}$ is given by

$$
\hat{\mathbf{J}}_{o}=\mathbf{T}_{p} \mathbf{T}_{S U(2)_{2}} \mathbf{T}_{s} \mathbf{T}_{S U(2)_{1}} \hat{\mathbf{J}}_{i} .
$$

The resultant intensity at the output of this system is

$$
I_{\text {out }}(t)=\eta I_{\text {in }}(t)\left|\tilde{r}_{1} \cos \theta_{1} \cos \theta_{2}-\tilde{r}_{2} \sin \theta_{1} \sin \theta_{2} e^{-i\left(2 \phi_{1}+2 \alpha_{2}\right)}\right|^{2},
$$


where $\theta_{1}, \theta_{2}, \phi_{1}$, and $\alpha_{2}$ are as defined in the previous section. In ellipsometry, there are two parameters that are of interest: $\psi$ and $\Delta$. The quantity $\psi$ is related to the magnitude of the ratio of the sample's eigenpolarization complex reflection coefficients, $\tilde{r}_{1}$ and $\tilde{r}_{2}$, via $\tan \psi=\left|\tilde{r}_{2} / \tilde{r}_{1}\right| ; \Delta$ is the phase shift between them. ${ }^{2}$ With this in mind, Eq. 5 can be re-written in terms of the ellipsometric parameters. The resultant equation is

$$
I_{\text {out }}(t)=\eta I_{\text {in }}(t)\left|\tilde{r}_{2}\right|^{2}\left|\tan \psi e^{i\left(\Delta+2 \phi_{1}+2 \alpha_{2}\right)} \cos \theta_{1} \cos \theta_{2}-\sin \theta_{1} \sin \theta_{2}\right|^{2} .
$$

From this one equation, many forms of classical ellipsometry can be understood. We will now examine the special cases of null and interferometric ellipsometry. In the traditional null ellipsometer ${ }^{2}$ the sample is illuminated with

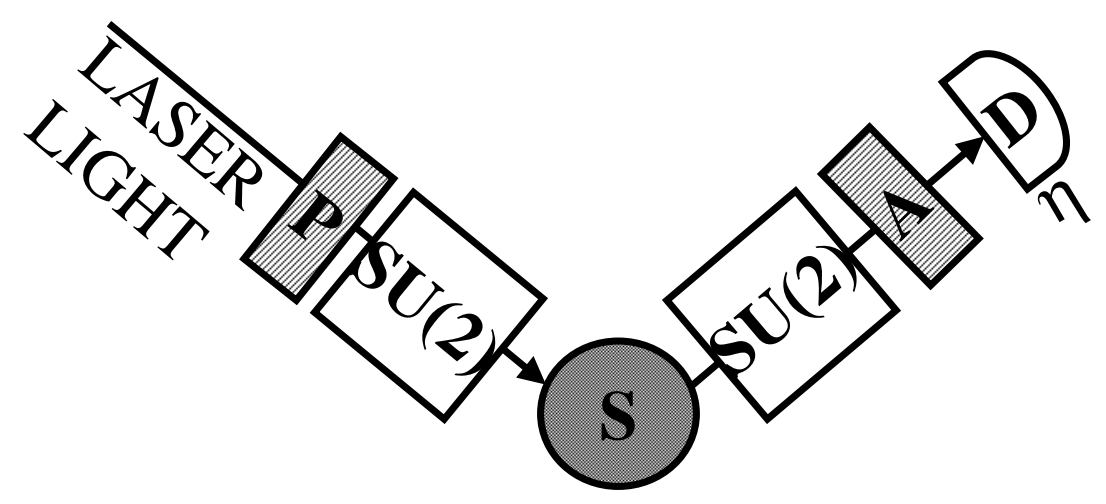

Figure 1. General ellipsometry. SU(2) represents the allowed polarization devices used, $\mathrm{P}$ a linear polarizer, A a linear polarization analyzer, and D an optical detector with efficiency $\eta$. The sample $\mathrm{S}$ is characterized by the ellipsometric parameters $\psi$ and $\Delta$ as defined in the text.

a beam of light that can be prepared in any state of polarization. The reflected light, which is generally elliptically polarized, is then analyzed. The polarization of the incident beam is adjusted to compensate for the change in the relative amplitude and phase, introduced by the sample, between the two eigenpolarizations, so that the resulting reflected beam is linearly polarized. If passed through an orthogonal linear polarizer, this linearly polarized beam will yield a null (zero) measurement at the optical detector. By setting $I_{\text {out }}(t)=0$ in Eq. 6, the two conditions for the null are

$$
\tan \theta_{1} \tan \theta_{2}=\tan \psi
$$

and

$$
\Delta=-2\left(\phi_{1}+\alpha_{2}\right) .
$$

It is clear from these conditions that the null ellipsometer requires neither a calibrated source nor a calibrated detector since it does not measure intensity, but instead records a null. It is also clear that null ellipsometry is essentially based on a single measurement, i.e., the location of the null, and as such, the accuracy of the measured ellipsometric parameters is very sensitive to the devices used in the apparatus. The purpose of the reference sample here is to calibrate the null, for example to find its initial location (the rotational axis of reference at which an initial null is obtained) and then to compare this with the subsequent location upon inserting the sample into the apparatus. Therefore, of the four aforementioned attributes, null ellipsometry fails to meet only the first that calls for no external reference sample.

Conventional interferometric ellipsometers that are constructed to be temporal interferometers do not satisfy at least three of the attributes for accurate measurements, mainly because the sample parameters cannot be separated from the parameters of the elements used to construct the interferometer. For polarization interferometers these attributes would be met were it not for the time-varying source intensity. However, in the presence of such a source, a beam splitter is required to perform normalization of the detected output intensity. As mentioned before, this process becomes noisy in the low-light regime and thus prevents accurate measurements 
from being obtained. It is worth mentioning here that for all interferometric ellipsometry schemes based on polarization interferometry, the third attribute requiring basis-independent measurements can be met by taking every combination of angle settings and extracting information about the ellipsometric parameters subsequently. This is achieved through automation but has the drawback of being slow. In the next section, we introduce an interferometric technique that obviates the need for the beam splitter while guaranteeing accurate ellipsometric measurements even at low light levels.

\section{QUANTUM ELLIPSOMETRY: CORRELATED-PHOTON ELLIPSOMETRY}

It has previously been shown that twin photons generated by the process of spontaneous parametric downconversion can be used in a coincidence-detection scheme to obtain absolute calibration of an optical detector. ${ }^{14,15}$ In this section, we demonstrate how the addition of the polarization degree of freedom allows accurate ellipsometric measurements from a sample to be obtained at low light levels.

In correlated-photon ellipsometry, a form of quantum ellipsometry, a laser (pump) beam illuminates a nonlinear optical crystal (NLC), in our case it is beta-barium borate, typically referred to as BBO. Quantum mechanics predicts that some of the pump photons disintegrate into pairs, known as signal and idler, which conserve energy (frequency-matching) and momentum (phase-matching). ${ }^{16,17}$ For our purposes, we choose the SPDC to be in a configuration known as 'type-I non-collinear'. 'Type-I' refers to the fact that the signal and idler photons have parallel polarizations; the term 'non-collinear' indicates that the signal and the idler photons are emitted in two different directions. The light at the output of the NLC is in a polarization-product state and is described by

$$
|\Psi\rangle=|\mathrm{HV}\rangle,
$$

where $\mathrm{H}$ and $\mathrm{V}$ represent horizontal and vertical polarizations, respectively. In one arm of the setup, the idler (signal) beam first passes through a linear polarizer $\mathrm{P}$ followed by an $\mathrm{SU}(2)$ element, and then reflects off the sample of interest before it encounters a second $\mathrm{SU}(2)$ element followed by a linear polarization analyzer A then single-photon photodetector $\mathrm{D}_{1}$. In the other arm, the signal (idler) beam is simply detected by single-photon photodetector $\mathrm{D}_{2}$. Generally, narrow-band interference filters are placed in front of each detector in order to select the degenerate-frequency photons. The detectors, two avalanche photodiodes operating in the Geiger mode, are part of a circuit that records the coincidence rate of photon pairs. The setup is shown in Fig. 2.

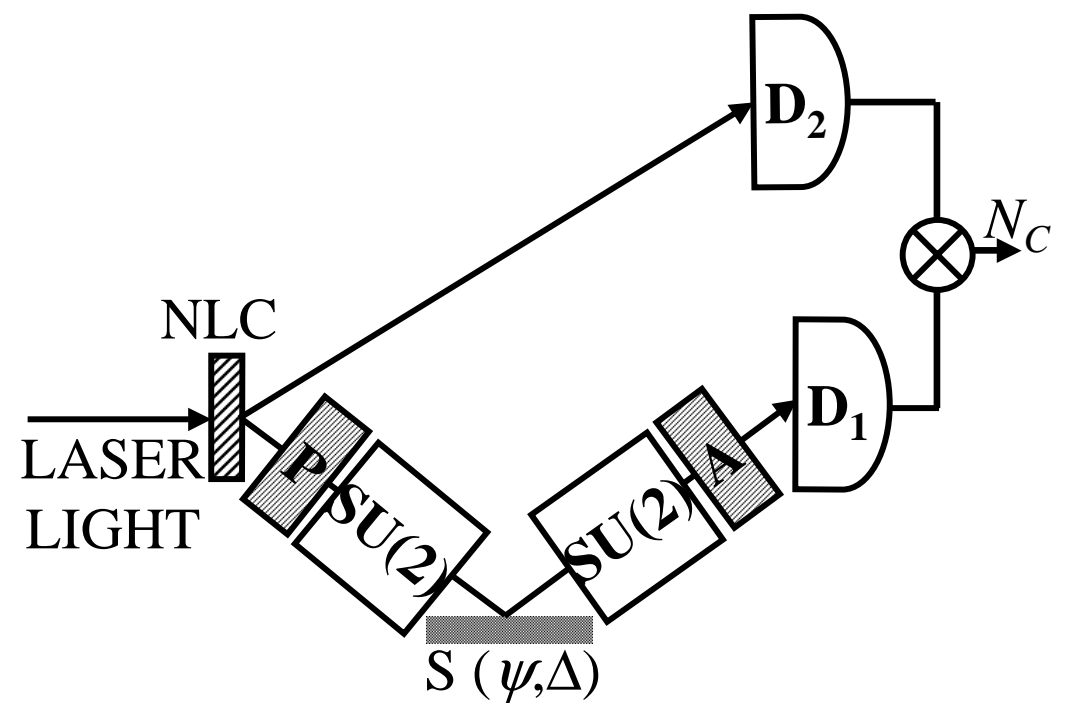

Figure 2. Correlated-photon ellipsometry. 
It can be shown that for the state described in Eq. (9), the obtained coincidence rate, $N_{c}$, at the detectors is given by

$$
N_{c}\left(\theta_{1}, \theta_{2}\right)=C I_{\text {in }} \eta_{1} \eta_{2}\left|\tilde{r}_{2}\right|^{2}\left|\tan \psi e^{i\left(\Delta+2 \phi_{1}+2 \alpha_{2}\right)} \cos \theta_{1} \cos \theta_{2}-\sin \theta_{1} \sin \theta_{2}\right|^{2},
$$

where $I_{\text {in }}$ is the pump intensity, the constant of proportionality $C$ includes the efficiency of downconversion, $\eta_{1}$ and $\eta_{2}$ are the quantum efficiencies of $\mathrm{D}_{1}$ and $\mathrm{D}_{2}$, respectively. Using this expression one can determine the ellipsometric parameters of the sample in a straightforward fashion simply by choosing different angle settings for $\theta_{1}$, for example, while scanning $\theta_{2}$. In this case the pump intensity is assumed to be constant in time but unknown to the observer. If the intensity of the pump is time varying, however, $I_{\text {in }}$ becomes $I_{\text {in }}(t)$ and the measurement protocol described above fails. To eliminate this problem, we have developed a procedure that employs an auxiliary measurement, the singles rate. This rate has been defined to be proportional to the intensity of the pump and the quantum efficiency of the detector. ${ }^{12}$ Let us define the singles rate for the sample-free optical path to be

$$
I_{2}=C I_{\text {in }}(t) \eta_{2} .
$$

By dividing the expression in Eq. (10) by that in Eq. (11), we obtain a normalized coincidence rate, $N_{n}$, given by

$$
N_{n}\left(\theta_{1}, \theta_{2}\right)=\frac{N_{c}\left(\theta_{1}, \theta_{2}\right)}{I_{2}}=\eta_{1}\left|\tilde{r}_{2}\right|^{2}\left|\tan \psi e^{i\left(\Delta+2 \phi_{1}+2 \alpha_{2}\right)} \cos \theta_{1} \cos \theta_{2}-\sin \theta_{1} \sin \theta_{2}\right|^{2} .
$$

To determine the ellipsometric parameters of the sample, we can choose the following strategy: for $\theta_{1}$ set to $90^{\circ}$, and $\theta_{2}$ scanned,

$$
N_{n}\left(90, \theta_{2}\right)=\eta_{1}\left|\tilde{r}_{2}\right|^{2} \sin ^{2} \theta_{2} .
$$

Similarly, for $\theta_{1}$ set to $0^{\circ}$, and $\theta_{2}$ scanned,

$$
N_{n}\left(0, \theta_{2}\right)=\eta_{1}\left|\tilde{r}_{2}\right|^{2} \tan ^{2} \psi \cos ^{2} \theta_{2} .
$$

From these equations it is clear that $\psi$ can be obtained by dividing the amplitude of Eq. (14) by that of Eq. (13). The resulting expression is

$$
\frac{N_{n}\left(0, \theta_{2}\right)}{N_{n}\left(90, \theta_{2}\right)}=\tan ^{2} \psi
$$

To determine $\Delta$ we divide the difference between measurements performed in a rectilinear basis, $N_{n}\left(45, \theta_{2}\right)$ and $N_{n}\left(-45, \theta_{2}\right)$, by the amplitude of Eq. (13). It can be shown that the resulting expression, $N_{\text {DIFF }}$, is

$$
N_{\mathrm{DIFF}}=\frac{\left[N_{n}\left(45, \theta_{2}\right)-N_{n}\left(-45, \theta_{2}\right)\right]}{N_{n}\left(90, \theta_{2}\right)}=-2 \cos \left(\Delta+2 \phi_{1}+2 \alpha_{2}\right) \tan \psi \cos \theta_{2} \sin \theta_{2}
$$

If we set the phases of the wave plates to zero, then $\Delta$ is directly obtained from the amplitude of Eq. (16). Therefore, the ellipsometric parameters are determined independently of a time-varying pump intensity and without knowledge of the values of the quantum efficiencies of the detectors used. It is not explicit in this analysis how this form of ellipsometry satisfies the requirement for basis-independent measurements, but since correlated-photon ellipsometry is a form of interferometric ellipsometry this attribute can be similarly met via automation. Furthermore, since quantum mechanics allows for a "perfect" copy of a beam to be created without a beam splitter, normalizing to eliminate noise sources inherent in the system is possible without introducing any errors. This is advantageous for low light levels, and is a feature unique to the quantum nature of correlatedphoton ellipsometry. We see from this analysis that correlated-photon ellipsometry meets all the attributes for accurate ellipsometric measurements.

\section{EXPERIMENTAL RESULTS}

Preliminary experiments have shown that quantum ellipsometry can be used to obtain values of $\psi$ and $\Delta$ that are comparable to those obtained from traditional ellipsometers. 
The actual experimental setup used to perform our measurements is a slightly-modified version of the one shown in Fig. 2. Specifically, the $\mathrm{SU}(2)$ element after the sample is removed while the one before the sample is chosen to be a linear polarization generator (LPG). Furthermore we redefine the angles $\theta_{1}$ and $\theta_{2}$ to be the angles of the axes of the LPG and polarization analyzer with respect to the horizontal direction, respectively. This way, the results from Eqns. (12)-(16) are directly applicable with the additional condition that $\phi_{1}$ and $\alpha_{2}$ are set to zero. A 351-nm $\mathrm{cw} \mathrm{Ar}{ }^{+}$laser pump illuminated a BBO crystal to produce degenerate twin photons centered at $702 \mathrm{~nm}$. Two avalanche photodiodes operating in the Geiger mode were used as detectors $\left(\mathrm{D}_{1}\right.$ and $\mathrm{D}_{2}$ ). Interference filters centered at $702 \mathrm{~nm}$ with $10 \mathrm{~nm}$ bandwidths were placed in front of each detector.

Using the procedures represented by Eqns. (13)-(16), ellipsometric data was obtained for a single-crystal Si sample oriented at an angle of incidence of $30^{\circ}$. The results are represented by the interferometric curves in Fig. 3 and Fig. 4. The experimentally-determined values for $\psi$ and $\Delta$ were compared to those obtained using a classical ellipsometer (specifically, a variable angle spectroscopic ellipsometer) at the J. A. Woollam Co., Inc. Using the classical ellipsometer, the measured values of $\psi$ and $\Delta$ were $40.4^{\circ}$ and $179.7^{\circ}$, respectively, with a film thickness of $27.8 \AA$. These values were also in accordance with calculations carried out using the appropriate Sellmeier dispersion formula. ${ }^{18,19}$ Using our correlated-photon ellipsometer, $\psi$ and $\Delta$ were determined to be $40.7 \pm .6^{\circ}$ and $168 \pm 2^{\circ}$, respectively.

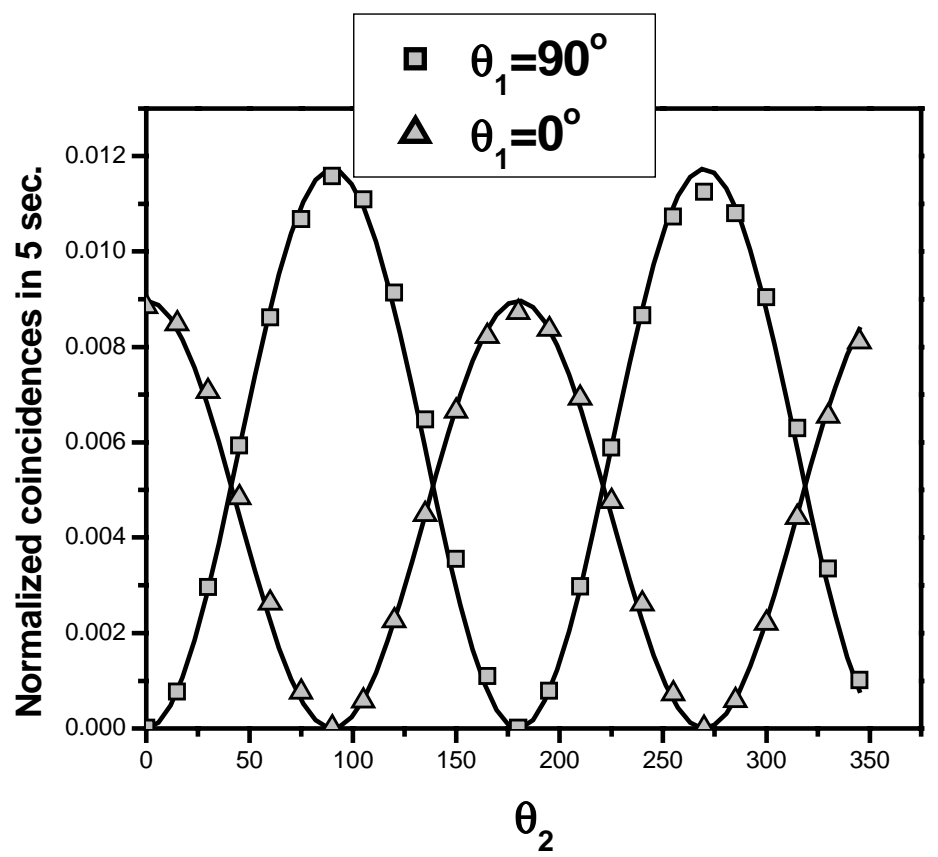

Figure 3. Coincidence interference patterns obtained by scanning the angle $\theta_{2}$, for $\theta_{1}$ fixed at either $0^{\circ}$ or $90^{\circ}$.

The difference between our experimentally-determined value for $\Delta$ and the expected value can be explained as follows: the values $0^{\circ}$ and $180^{\circ}$ are considered regions of uncertainty for $\Delta$ because of the flat dependence of $\Delta$ at these values. The closer the expected value of $\Delta$ is to these values, the less accurate the measurements are. This is a well-known problem in classical ellipsometry that is typically dealt with by inserting a quarter-wave plate to add a $90^{\circ}$ phase shift to the measured value of $\Delta .^{3}$

\section{CONCLUSION}

Classical ellipsometric measurements are limited in their accuracy by virtue of the need for calibration by an external reference sample or because of unavoidable noise fluctuations that dominate in the low-light regime. 


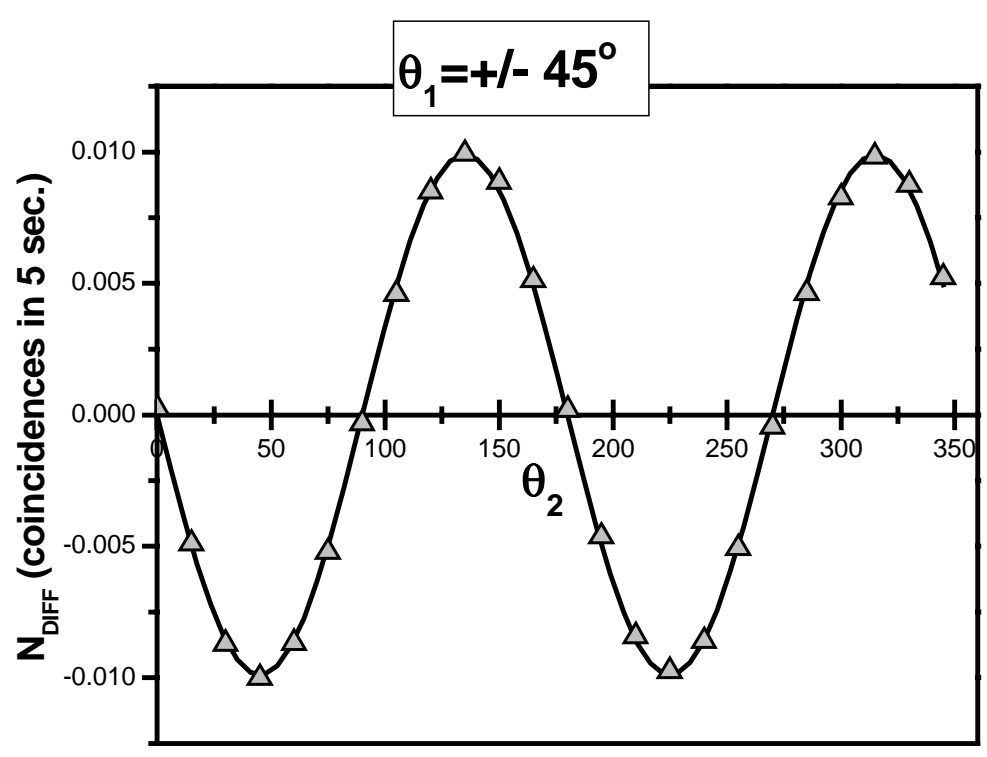

Figure 4. Coincidence interference pattern determined by scanning the angle $\theta_{2}$ in accordance with Eq. 16.

Quantum ellipsometry mitigates this limitation by using correlated-photon pairs created by the SPDC process, in conjunction with a coincidence-counting detection scheme.

Preliminary experimental results for $\psi$ and $\Delta$ for a Si sample are reported. The measured value of $\psi$ was in good agreement with the expected value, but the discrepancy between the measured and expected value for $\Delta$ was larger. We believe that this problem is associated with our inability to resolve $\Delta$ for values of $0^{\circ}$ or $180^{\circ}$. This can be corrected by inserting a quarter-wave plate in the system in order to move $\Delta$ away from these indiscriminate regions.

Since the SPDC source is inherently broadband, narrowband spectral filters must be used to ensure that the ellipsometric data are measured at a specific frequency. Spectroscopic data can be obtained by employing a bank of such filters. Alternatively, techniques from Fourier-transform spectroscopy may be used to directly make use of the broadband nature of the source in ellipsometric measurements.

\section{ACKNOWLEDGMENTS}

This work was supported by the Gates Millennium Scholars Program, the National Science Foundation, and by the Center for Subsurface Sensing and Imaging Systems (CenSSIS), an NSF engineering research center. We extend special thanks to Ron Synowicki and the staff at J.A. Woollam Co., Inc. for carrying out measurements using their VASE ellipsometer.

\section{REFERENCES}

1. P. Drude, "Bestimmung optischer Konstanten der Metalle," Ann. d. Physik u. Chemie 39, pp. 481-554, 1890.

2. R. M. A. Azzam and N. M. Bashara, Ellipsometry and Polarized Light, North-Holland, Amsterdam, 1977.

3. H. G. Tompkins and W. A. McGahan, Spectroscopic Ellipsometry and Reflectometry, Wiley, New York, 1999.

4. A. Rothen, "The ellipsometer, an apparatus to measure thicknesses of thin surface films," Rev. Sci. Instrum. 16, pp. 26-30, 1945. 
5. A. B. Winterbottom, "Optical methods of studying films on reflecting bases depending on polarization and interference phenomena," Trans. Faraday Society 42, pp. 487-495, 1946.

6. M. Mansuripur, "Ellipsometry," Opt. \& Phot. News 11 (4), pp. 52-56, 2000.

7. M. M. Hayat, A. Joobeur, and B. E. A. Saleh, "Reduction of quantum noise in transmittance estimation using photon-correlated beams," J. Opt. Soc. Am. A 16, pp. 348-358, 1999.

8. A. F. Abouraddy, K. C. Toussaint, Jr., A. V. Sergienko, B. E. A. Saleh, and M. C. Teich, "Ellipsometric measurements by use of photon pairs generated by spontaneous parametric down-conversion," Opt. Lett. 26, pp. 1717-1719, 2001.

9. A. F. Abouraddy, K. C. Toussaint, Jr., A. V. Sergienko, B. E. A. Saleh, and M. C. Teich, "Entangled-photon ellipsometry," J. Opt. Soc. Am. B 19, pp. 656-662, 2002.

10. C. H. Bennett, G. Brassard, C. Crepeau, R. Jozsa, A. Peres, and W. K. Wootters, "Teleporting an unknown quantum state via dual classical and Einstein-Podolsky-Rosen channels," Phys. Rev. Lett. 70, pp. 1895-1899, 1993.

11. A. K. Ekert, J. G. Rarity, P. R. Tapster, and G. M. Palma, "Practical quantum cryptography based on two-photon interferometry," Phys. Rev. Lett. 69, pp. 1293-1295, 1992.

12. D. N. Klyshko, "Utilization of vacuum fluctuations as an optical brightness standard," Sov. J. Quantum Electron. 7, p. 591, 1977.

13. D. Branning, A. L. Migdall, and A. V. Sergienko, "Simultaneous measurement of group and phase delay between two photons," Phys. Rev. A 62, p. 063808, 2000.

14. D. N. Klyshko, "Use of two-photon light for absolute calibration of photoelectric detectors," Kvantovaya Elektron 7, pp. 1932-1940, 1980. [Sov. J. Quantum Electron. 10(9), 1112-1116 (1980)].

15. A. Migdall, R. Datla, A. V. Sergienko, and Y. H. Shih, "Absolute detector quantum efficiency measurements using correlated photons," Metrologia 32, pp. 479-483, 1995.

16. D. N. Klyshko, Photons and Nonlinear Optics, ch. 1 and 6. Nauka, Moscow, 1980. [Translation: Gordon and Breach, 1988].

17. B. E. A. Saleh and M. C. Teich, Fundamentals of Photonics, Wiley, New York, 1991.

18. E. D. Palik, ed., Handbook of Optical Constants of Solids, Academic Press, New York, 1985.

19. E. D. Palik, ed., Handbook of Optical Constants of Solids III, Academic Press, New York, 1995. 